ИЗВЕСТИЯ АКАДЕМИИ НАУК ЭСТОНСКОЙ ССР. ТОМ 27 ФИЗИКА * МАТЕМАТИКА. 1978, ㄴ 4

\title{
О ВНУТРИСЛОИНОМ КРИЗИСЕ ТЕПЛООТДАЧИ ПРИ КИПЕНИИ НА ПОВЕРХНОСТИ, ПОКРЫТОЙ ПОРИСТЫМ МАТЕРИАЛОМ
}

\author{
(Представлена И. Эпиком)
}

Условия теплоотдачи при кипении жидкости на поверхности с пористым покрытием определяются условиями парообразования, а также условиями течения пара и жидкости в пористом слое $\left[{ }^{1-5}\right]$. Существенное влияние на эти процессы оказывают структурные характеристики покрытия - толщина, пористость и распределение пор по размерам. Механизм кипения в первом приближении можно представить как испарение жидкости с кривых менисков раздела фаз внутри пористого материала и удаление пара через сравнительно большие поры, где капиллярное давление жидкости низкое. В пористый материал жидкость впитывается по мелким порам под действием капиллярных сил. При этом предполагается полное смачивание.

Тепловой поток с поверхности нагрева идет к поверхности раздела фаз путем теплопроводности через скелет пористого материала и жидкость, вызывая изменение агрегатного состояния теплоносителя. Межфазная поверхность между жидкостью и паром внутри пористого слоя имеет при кипении сложную конфигурацию, зависящую от структуры пористого материала и величины тепловой нагрузки.

Примем для простоты, что испарение жидкости в пористом слое происходит в параллельной поверхности нагрева прослойке материала малой толщины. Тогда по величине тепловой нагрузки процесс кипения можно разделить на три режима. В первом поверхность испарения непосредственно соприкасается с поверхностью нагрева под пористым слоем, во втором она отделяется от поверхности нагрева слоем сухого пористого материала (внутрислойный кризис) и в третьем отделяегся от пористого материала слоем пара (кризис кипения). Қаждому режиму соответствуют свои тепловые нагрузки.

Первый режим продолжается до тех пор, пока сила суммарного сопротивления пористого слоя течению через него жидкости и пара не превысит силы максимального капиллярного давления, создаваемого менисками жидкости в порах. Когда это условие нарушится, т. е. когда сопротивление пористого слоя увеличится настолько, что жидкость уже не сможет достичь поверхности нагрева, начнется второй режим.

Для определения зависимости тепловой нагрузки, соответствующей 
началу внутрислойного кризиса, от структуры пористого материала рассмотрим поликапиллярную модель пористого тела, представив поры прямыми цилиндрическими капиллярами с различными радиусами и длиной, равной толщине пористого слоя. Для этой модели важной характеристикой является распределение пор по размерам. Дифференциальная кривая распределения

$$
f(a)=\frac{d V}{d a}
$$

описывает скорость изменения относительного объема пор с изменением радиуса. В реальном пористом материале это распределение носит непрерывный характер и отражает тот факт, что проницаемость пористого покрытия для жидкости и пара находится в прямой зависимости от тепловой нагрузки. Хотя кривая дифференциального распределения пор по радиусам имеет сложную форму, ради упрощения задачи представим ее состоящей только из двух пиков - жидкостных пор радиусом $a_{L}$, которые занимают площадь удельного сечения пор $A_{L}$, и паровых пор радиусом $a_{V}$, которые занимают площадь удельного сечения пор $A_{V}$. При этом $a_{L}<a_{V \text { и }}$

$$
A_{L}+A_{V}=\varepsilon
$$

где $\varepsilon$ - открытая пористость.

Исходя из такой модели пористого слоя, определим оптимальное соотношение размеров паровых и жидкостных пор, воспользовавшись зависимостью, полученной применительно к тепловым трубам [6]:

$$
a_{L}^{2}=a_{V}^{2} \frac{A_{V} v_{L}}{v_{V} A_{L}}
$$

где $v-$ кинематическая вязкость.

Выполнение условия (3) означает, что сопротивление течению жидкости и сопротивление течению пара в пористом слое одинаковы и равняются половине капиллярной тяги $\Delta p$ :

$$
\Delta p=2 \sigma / a_{L},
$$

где $\sigma-$ поверхностное натяжение.

Используя аналогию с тепловой трубой, находим тепловую нагрузку $q_{*}$, соответствующую началу отделения зоны испарения от поверхности нагрева, т. е. началу внутрислойного кризиса $\left[{ }^{6}\right]$ :

$$
q_{*}=\frac{\sigma r A_{V} a_{V}^{2}}{4 v_{V} a_{L} L},
$$

где $r$ - скрытая теплота парообразования, $L$ - толщина пористого слоя.

Для иллюстрации влияния распределения пор на эту нагрузку на рисунке приведена зависимость

$$
q=f\left(A_{V}, A_{L}, \frac{a_{L}}{a_{V}}\right)
$$

в случае кипения трифтортрихлорэтана (Ф-113) при $A_{L}+A_{V}=0,6$; $L=10^{-3} \mu ; a_{V}=6 \cdot 10^{-5} \mu$. Видно, что с увеличением пористости и раз- 
меров пор $q *$ растет и достигает максимума при определенном соотношении радиусов жидкостных и паровых пор. Положение максимума зависит от кинематической вязкости жидкости и пара. В нашем случае q. имеет максимум при $a_{L} / a_{V}=0,55$ и $A_{L}=$ $=A_{V}$.

Размеры пор определяются как условиями парообразования, так и условиями транспорта жидкости и пара в пористом слое. С точки зрения теплоотдачи выгодно располагать высокой проницаемостью покрытия и низким тепловым сопротивлением фазового перехода. Однако эти требования противоречивы. Перепад между температурами на монолитной поверхности нагрева и кипящей жидкости можно представить суммой из двух слагаемых

$$
\Delta T=\Delta T_{1}+\Delta T_{2},
$$

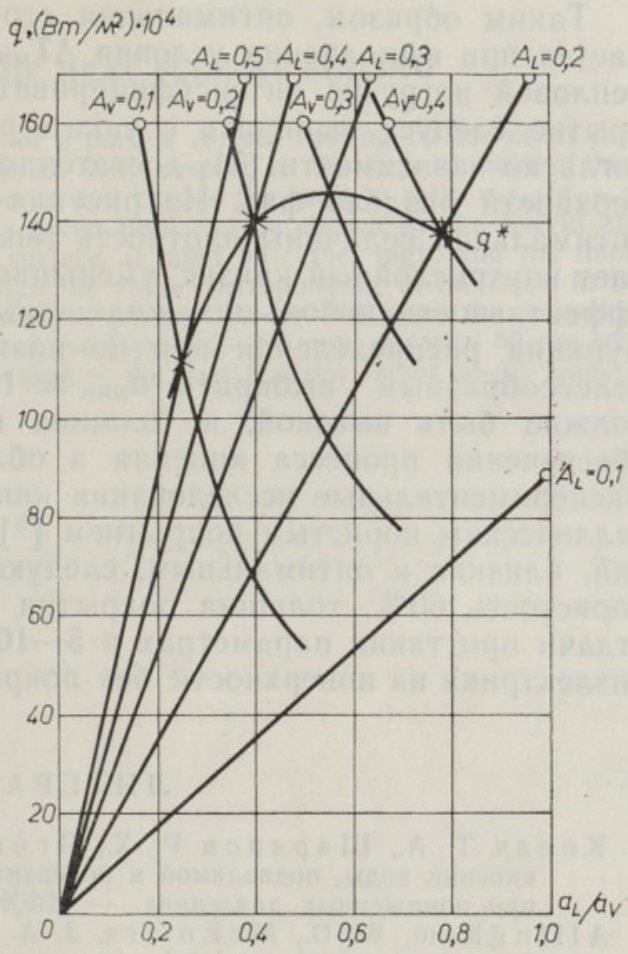

где $\Delta T_{1}-$ разность температур между поверхностью нагрева и жидкостью на кривой межфазной поверхности, $\Delta T_{2}$ - то же между жидкостью и паром над вогнутым мениском.

Увеличение размеров пор при неизменной пористости уменьшает удельную поверхность пористого покрытия обратно пропорционально радиусу пор. Следовательно, с ростом последних $\Delta T_{1}$ будет увеличиваться, так как $\Delta T_{1} \sim Q / S$, где $Q-$ тепловой поток, $S$ - удельная внутренняя поверхность, а $\Delta T_{2}-$ уменьшаться, поскольку увеличение радиуса пор повысит проницаемость пористого слоя и уменьшит необходимый для испарения перегрев жидкости с кривой поверхности. Это видно из следующих зависимостей. Для поликапиллярной модели при действительности закона Пуайзеля проницаемость определяется выражением

$$
k=\frac{\varepsilon}{8} \int_{a_{\mathrm{M} и}}^{a_{\mathrm{M} \mathrm{акс}}} a^{2} f(a) d a .
$$

Необходимый для испарения в пузырек сферической формы перегрев жидкости относительно пара в соответствии с законами Кельвина и Клапейрона будет равен

$$
\Delta T_{2}=\frac{2 \sigma T^{\prime \prime}}{a r_{Q V}}
$$

где $T^{\prime \prime}$ - температура насыщения над плоской поверхностью раздела, Qv - плотность пара. 
Таким образом, оптимальная структура пористого покрытия получается при выполнении условия $\Delta T_{\text {мин }}=\Delta T_{1}+\Delta T_{2}$. Чтобы при данной тепловой нагрузке интенсифицировать испарительное охлаждение, покрытие следует выбирать с таким размером пор, который обеспечит, согласно зависимости (7), достаточно малый перегрев жидкости на поверхности раздела фаз. Из рисунка видно, что при выборе $a_{L}$ ниже оптимальной величины плотность теплового потока, при которой наступает внутрислойный кризис, уменьшается. Это свидетельствует о низкой эффективности небольших жидкостных пор. Исходя из непрерывности функции распределения пор по размерам и рисунка, представляется

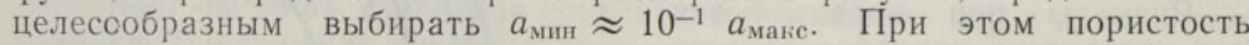
должна быть высокой, а толщина покрытия меньше требуемой для обеспечения процесса кипения в области тепловых нагрузок $q<q$ *. Экспериментальные исследования кипения Ф-113 на поверхности с металлическим пористым покрытием $\left[{ }^{5}\right]$ показали, что параметры покрытий, близкие к оптимальным, следующие: средний радиус пор $10^{-5} \mathrm{M}$, пористость $60 \%$, толщина покрытия 0,5-1,0 мм. Коэффициент теплоотдачи при таких параметрах в 5-10 раз выше, чем в случае кипения диэлектрика на поверхности без покрытия.

\section{Л И Т Е Р А Т У Р А}

1. Колач Т. А., Шарипов Р. Х., Ягов В. В. Исследование теплообмена при кипении воды, подводимой к поверхности нагрева капиллярно-пористым телом при пониженных давлениях. - ИФЖ, 1968, т. 11, № 6, с. 957-982.

2. Allingham, W. D., McEntire, J. A. Determination of boiling film coefficient for a heated horizontal tube in water-saturated wick material. - Trans. ASME, Ser. C, 1961 , v. $83, \mathrm{~N} 1$, p. $71-76$.

3. Costello, C. P., Frea, W. J. The roles of capillary wicking and surface deposits in the attainment of high pool boiling burnout. - AIChE Journal, 1964, v. 10, N 3, p. 393-398.

4. Czikk, A. M., G ottz m a n n, C. F. Performance of advanced heat transfer tubes in refrigerant flooded liquid coolers. - ASHRAE Journal, 1970, v. 76, N 1, p. $96-108$.

5. Кальчинский М. 3., Техвер Я. Х., Туник А. Т., Р айченко А. И., Медведенко А. Ф., Валликиви А. Ю., Л евин а Д. А. Свойства пористых материалов на основе алюминия, предназначенных для систем испарительного охлаждения электрических устройств. - В кн.: Materiały proszkowe u maszynach elektrycznych. Wroclaw, 1976, c. 35-41.

6. S chindler, M. Theoretisches Modell zum Wärmetransport in Wärmeübertragungsrohren. - Atomkernenergie, 1965, Bd. 10, N 9/10, S. 395-398.

Институт термофизики и электрофизики Академии наук Эстонской ССР
Поступила в редакцию 11/XI 1977

\section{J. TEHVER, A. TUNIK}

\section{SOOJUSULEKANDE KIHISISENE KRIIS POORSE KATTEGA PINNAL TOIMUVA KEEMISE KORRAL}

Artiklis on analüüsitud poorse pinnakatte struktuuri mõju kihisisesele kriisile dielektriliste vedelike keemisel vaba konvektsiooni korra!. 


\section{J. TEHVER, A. TUNIK}

\section{ON IN-COATING CRISIS OF HEAT FLUX AT BOILING}

In this paper the influence of the structure of a porous surface coating on the crisis in a layer for boiling dielectric fluids is considered. With respect to the improvement of heat transfer, it is suitable to avoid too small pores and choose the radii in ratio $a_{\max } / a_{\min } \approx$ $\approx 10$. The maximum radius for a pore in a given fluid can be chosen by the Kelvin and Clapeyron laws. As to the porosity of the surface, it must be rather high, and the thickness of the porous layer must be smaller than the value corresponding to the crisis in the layer. The experimentally obtained close optimum parameters for F-113 are the following: medium radius of a pore $10 \mu \mathrm{m}$, thickness of the layer $0.5-1.0 \mathrm{~mm}$, porosity 60 per cent. The given coating permits to increase the value of the heat transfer factor $5-10$ times as compared to a smooth surface. 